\title{
Optimal Jamming of Wireless Localization Systems
}

\author{
Sinan Gezici*, Mohammad Reza Gholami ${ }^{\natural}$, Suat Bayram ${ }^{\natural}$ and Magnus Jansson ${ }^{\natural}$ \\ * Department of Electrical and Electronics Engineering, Bilkent University, 06800, Ankara, Turkey \\ $\natural$ ACCESS Linnaeus Center, Electrical Eng., KTH Royal Institute of Technology, 100 44, Stockholm, Sweden \\ b Department of Electrical and Electronics Engineering, Turgut Ozal University, 06010, Ankara, Turkey \\ Emails: gezici@ee.bilkent.edu.tr, mohrg@kth.se, sbayram@turgutozal.edu.tr, janssonm@kth.se
}

\begin{abstract}
In this study, optimal jamming of wireless localization systems is investigated. Two optimal power allocation schemes are proposed for jammer nodes in the presence of total and peak power constraints. In the first scheme, power is allocated to jammer nodes in order to maximize the average Cramér-Rao lower bound (CRLB) of target nodes whereas in the second scheme the power allocation is performed for the aim of maximizing the minimum CRLB of target nodes. Both schemes are formulated as linear programs, and a closed-form expression is obtained for the first scheme. Also, the full total power utilization property is specified for the second scheme. Simulation results are presented to investigate performance of the proposed schemes.
\end{abstract}

Keywords: Localization, jammer, power allocation, Cramér-Rao lower bound.

\section{INTRODUCTION}

Over the last two decades, wireless localization has not only become an important application for various systems and services, but also drawn significant interest from research communities [1]-[3]. Among various applications of wireless localization are inventory tracking, home automation, tracking of robots, fire-fighters and miners, patient monitoring, and intelligent transport systems [4]. In order to realize such applications under certain accuracy requirements, both theoretical and experimental studies have been performed in the literature (e.g., [5], [6]).

Although various studies have been performed on wireless localization, jamming of wireless localization systems has not been investigated in detail. In the literature, there exist some studies on GPS jamming and antijamming, such as [7]-[9]. However, for a given wireless localization system, a general theoretical analysis that quantifies the effects of jamming on localization accuracy has not been performed, and optimal jamming strategies have not been investigated before. In this study, a theoretical framework is proposed for jamming of wireless localization systems. In the proposed framework, the aim of a wireless localization system is, as usual, to estimate

\footnotetext{
${ }^{0}$ This work was supported in part by the European Commission in the framework of the FP7 Network of Excellence in Wireless COMmunications NEWCOM \# (contract no. 318306). S. Gezici's research was also supported in part by the Distinguished Young Scientist Award of Turkish Academy of Sciences (TUBA-GEBIP 2013).
}

positions of target nodes based on signal exchanges with anchors nodes, which have known positions, while the aim of jammer nodes is to degrade the performance (accuracy) of the wireless localization system as much as possible. A mathematical formulation is obtained for the proposed framework in terms of the optimization of theoretical limits, namely, the Cramér-Rao lower bound (CRLB). Two optimal power allocation schemes are proposed for jammer nodes under total and peak power constraints. In the first scheme, the optimal power allocation is performed for jammer nodes in order to maximize the average CRLB of the target nodes whereas in the second scheme the aim is to maximize the minimum CRLB of the target nodes. For both schemes, the optimization problems are formulated as linear programs, and a closedform solution is obtained for the first scheme. In addition, the properties of the optimal solution are characterized for the second scheme. Simulations are performed in order to illustrate the effectiveness of the proposed approaches.

Although there exists no previous work on optimal power allocation for jammer nodes in a wireless localization system, power allocation for wireless localization and radar systems has recently been considered in [10]-[14]. In [10], optimal transmit power allocation is performed for anchor nodes in order to minimize the squared position error bound (SPEB) and the maximum directional position error bound (mDPEB) of the localization system. Conic programming is employed for efficient solutions, and improvements over uniform power allocation are illustrated. In [12], the optimal power allocation strategies are investigated for target localization in distributed multiple-radar system, where the total transmit power and the CRLB are considered as the two metrics in the optimization problems. In [13], ranging energy optimization is studied for a wireless localization system that performs two-way ranging between a target node and anchor nodes by considering a specific accuracy requirement in a service area. The joint power and bandwidth allocation problem for wireless localization systems is formulated in [15], and the resulting non-convex problem is solved approximately based on Taylor expansion, and iterative optimization of power and bandwidth separately.

The main contributions of this study can be summa- 
rized as follows:

- Optimal power allocation strategies are investigated for jammer nodes in a wireless localization system for the first time.

- Two optimal power allocation schemes are developed for jammer nodes in order to maximize the average or the minimum of the CRLBs for target nodes. Both schemes are formulated as linear programs.

- A closed-form solution is obtained for the scheme that maximizes the average CRLB.

- It is shown that the scheme that maximizes the minimum CRLB utilizes the all available total power.

The remainder of the paper is organized as follows. In Section II, the system model is introduced. In Section III, two power allocation formulations are proposed for optimal jamming of wireless localization systems, and the optimal power allocation schemes are characterized. Simulation results are presented in Section IV, and the concluding remarks are made in Section V.

\section{System Model}

Consider a wireless localization system consisting of $N_{A}$ anchor nodes and $N_{T}$ target nodes located at $\boldsymbol{y}_{i} \in$ $\mathbb{R}^{2}, i=1, \ldots, N_{A}$ and $\boldsymbol{x}_{i} \in \mathbb{R}^{2}, i=1, \ldots, N_{T}$, respectively. ${ }^{1}$ It is assumed that the target nodes estimate their locations based on received signals from the anchor nodes, which have known locations; that is, self-positioning is considered [4]. In addition to the target and anchor nodes, there exist $N_{J}$ jammer nodes at $\boldsymbol{z}_{i} \in \mathbb{R}^{2}, i=1, \ldots, N_{J}$ in the system, which aim to degrade the localization performance of the system. The jammer nodes are modeled to transmit Gaussian noise in accordance with the common approach in the literature [16]-[18]. An example of the proposed system model is shown in Fig. 1, where there are four anchor nodes $\left(N_{A}=4\right)$, three target nodes $\left(N_{T}=3\right)$, and three jammer nodes $\left(N_{J}=3\right)$.

In this study, non-cooperative localization is considered; that is, target nodes are assumed to receive signals only from anchor nodes (i.e., not from other target nodes) for localization purposes. In addition, the connectivity sets are defined as $\mathcal{A}_{i} \triangleq\{j \in$ $\left\{1, \ldots, N_{A}\right\} \mid$ anchor node $j$ is connected to target node $\left.i\right\}$ for $i \in\left\{1, \ldots, N_{T}\right\}$. Then, the received signal at target node $i$ coming from anchor node $j$ can be expressed as

$r_{i j}(t)=\sum_{k=1}^{L_{i j}} \alpha_{i j}^{k} s\left(t-\tau_{i j}^{k}\right)+\sum_{\ell=1}^{N_{J}} \gamma_{i \ell} \sqrt{P_{\ell}^{J}} v_{i \ell}(t)+n_{i j}(t)$

for $t \in\left[0, T_{\mathrm{obs}}\right], i \in\left\{1, \ldots, N_{T}\right\}$ and $j \in \mathcal{A}_{i}$, where $T_{\mathrm{obs}}$ is the observation time, $\alpha_{i j}^{k}$ and $\tau_{i j}^{k}$ denote, respectively, the amplitude and delay of the $k$-th multipath component

\footnotetext{
${ }^{1}$ The generalization to the three-dimensional scenario is straightforward, but is not explored in this study.
}

between anchor node $j$ and target node $i, L_{i j}$ is the number of paths between target node $i$ and anchor node $j$, and $\gamma_{i \ell}$ represents the channel coefficient between target node $i$ and the $\ell$-th jammer node, which has a transmit power of $P_{\ell}^{J}$. The transmit signal $s(t)$ is known and the measurement noise $n_{i j}(t)$ and the jammer noise $\sqrt{P_{\ell}^{J}} v_{i \ell}(t)$ are assumed to be independent zero-mean white Gaussian random processes, where the average power of $n_{i j}(t)$ is $N_{0} / 2$ and that of $v_{i \ell}(t)$ is equal to one. The delay $\tau_{i j}^{k}$ is given by

$$
\tau_{i j}^{k} \triangleq \frac{\left\|\boldsymbol{y}_{j}-\boldsymbol{x}_{i}\right\|+b_{i j}^{k}}{c}
$$

with $b_{i j}^{k} \geq 0$ denoting a range bias and $c$ being the speed of propagation. Set $\mathcal{A}_{i}$ is partitioned as

$$
\mathcal{A}_{i} \triangleq \mathcal{A}_{i}^{L} \cup \mathcal{A}_{i}^{N L}
$$

where $\mathcal{A}_{i}^{L}$ and $\mathcal{A}_{i}^{N L}$ represent the sets of anchors nodes with line-of-sight (LOS) and non-line-of-sight (NLOS) connections to target node $i$, respectively.

\section{Optimal Power Allocation for Jammer NODES}

In this section, the aim is to obtain optimal power allocation strategies for the jammer nodes in order to minimize the localization performance of the system. Two different optimization criteria are considered in terms of the average and the minimum CRLB for the target nodes. To that aim, we first present the CRLB expressions for the target nodes.

\section{A. CRLB for Location Estimation of Target Nodes}

To specify the set of unknown parameters related to target node $i$, the following vector is defined, which consists of the bias terms in the LOS and NLOS cases:

$$
\boldsymbol{b}_{i j}= \begin{cases}{\left[b_{i j}^{2} \ldots b_{i j}^{L_{i j}}\right]^{T},} & \text { if } j \in \mathcal{A}_{i}^{L} \\ {\left[b_{i j}^{1} \ldots b_{i j}^{L_{i j}}\right]^{T},} & \text { if } j \in \mathcal{A}_{i}^{N L}\end{cases}
$$

Based on (4), the unknown parameters related to target node $i$ are defined as [19]

$$
\boldsymbol{\theta}_{i} \triangleq\left[\begin{array}{llll}
\boldsymbol{x}_{i}^{T} & \boldsymbol{b}_{i \mathcal{A}_{i}(1)}^{T} & \cdots & \boldsymbol{b}_{i \mathcal{A}_{i}\left(\left|\mathcal{A}_{i}\right|\right)}^{T}
\end{array}\right]^{T},
$$

where $\mathcal{A}_{i}(j)$ denotes the $j$-th element of set $\mathcal{A}_{i}$ and $\left|\mathcal{A}_{i}\right|$ denotes the number of elements in $\mathcal{A}_{i}$.

The CRLB, which provides a lower bound on the variance of any unbiased estimator, for location estimation is given by [20]

$$
\mathbb{E}\left\{\left\|\hat{\boldsymbol{x}}_{i}-\boldsymbol{x}_{i}\right\|^{2}\right\} \geq \operatorname{tr}\left\{\left[\boldsymbol{F}_{i}^{-1}\right]_{2 \times 2}\right\},
$$

where $\hat{\boldsymbol{x}}_{i}$ denotes an unbiased estimate of the location of target node $i$, tr represents the trace operator, and $\boldsymbol{F}_{i}$ 
is the Fisher information matrix for vector $\boldsymbol{\theta}_{i}$. Following the steps taken in [5], $\left[\boldsymbol{F}_{i}^{-1}\right]_{2 \times 2}$ can be expressed as

$$
\left[\boldsymbol{F}_{i}^{-1}\right]_{2 \times 2}=\boldsymbol{J}_{i}\left(\boldsymbol{x}_{i}, \boldsymbol{p}^{J}\right)^{-1}
$$

where the equivalent Fisher information matrix $\boldsymbol{J}_{i}\left(\boldsymbol{x}_{i}, \boldsymbol{p}^{J}\right)$ in the absence of prior information about the location of the target node is calculated as

$$
\boldsymbol{J}_{i}\left(\boldsymbol{x}_{i}, \boldsymbol{p}^{J}\right)=\sum_{j \in \mathcal{A}_{i}^{L}} \frac{\lambda_{i j}}{N_{0} / 2+\boldsymbol{a}_{i}^{T} \boldsymbol{p}^{J}} \boldsymbol{\phi}_{i j} \boldsymbol{\phi}_{i j}^{T}
$$

with

$$
\begin{aligned}
\lambda_{i j} & \triangleq \frac{4 \pi^{2} \beta^{2}\left|\alpha_{i j}^{1}\right|^{2} \int_{-\infty}^{\infty}|S(f)|^{2} d f}{c^{2}}\left(1-\xi_{j}\right), \\
\boldsymbol{a}_{i} & \triangleq\left[\left|\gamma_{i 1}\right|^{2} \cdots\left|\gamma_{i N_{J}}\right|^{2}\right]^{T}, \\
\boldsymbol{p}^{J} & \triangleq\left[P_{1}^{J} \cdots P_{N_{J}}^{J}\right]^{T}, \\
\boldsymbol{\phi}_{i j} & \triangleq\left[\begin{array}{ll}
\cos \varphi_{i j} & \sin \varphi_{i j}
\end{array}\right]^{T} .
\end{aligned}
$$

In (9), $\beta$ is the effective bandwidth, which is expressed as

$$
\beta=\sqrt{\frac{\int_{-\infty}^{\infty} f^{2}|S(f)|^{2} d f}{\int_{-\infty}^{\infty}|S(f)|^{2} d f}},
$$

with $S(f)$ denoting the Fourier transform of $s(t)$, and the path-overlap coefficient $\xi_{j}$ is a non-negative number between zero and one, i.e., $0 \leq \xi_{j} \leq 1$ [10]. Also, in (12), $\varphi_{i j}$ denotes the angle between target node $i$ and anchor node $j$. In addition, it is assumed that the elements of $\boldsymbol{a}_{i}$ are non-zero (i.e., strictly positive) for $i \in\left\{1,2, \ldots, N_{T}\right\}$.

Remark 1: In this study, the jammer nodes are assumed to know the locations of the anchor and target nodes and the channel gains. In practice, this information may not be available to jammer nodes completely. However, this assumption is employed in this study for two purposes: (i) to obtain initial results that can form a basis for future studies on the problem of optimal power allocation of jammer nodes in localization systems (which has not been studied before), (ii) to provide theoretical limits on the best achievable performance of jammer nodes; that is, if the jammer nodes are smart and can learn all the environmental parameters, the localization accuracy obtained in this study can be achieved; otherwise, the localization accuracy is bounded by the obtained results.

\section{B. Optimal Power Allocation Strategies}

Before the introduction of the proposed optimal power allocation strategies, the dependence of the CRLB for target node $i$ (that is, the trace of $\boldsymbol{J}_{i}\left(\boldsymbol{x}_{i}, \boldsymbol{p}^{J}\right)^{-1}$ in (7)) on the power vector of the jammer nodes, $\boldsymbol{p}^{J}$, is specified.

Lemma 3.1: Consider the equivalent Fisher information matrix in (8). The trace of the inverse of $\boldsymbol{J}_{i}\left(\boldsymbol{x}, \boldsymbol{p}^{\boldsymbol{J}}\right)$ is an affine function with respect to $\boldsymbol{p}^{J}$.
Proof: From the definition of the equivalent Fisher information matrix in (8), it can be shown that

$$
\begin{aligned}
\operatorname{tr} & \left\{\boldsymbol{J}_{i}\left(\boldsymbol{x}_{i}, \boldsymbol{p}^{J}\right)^{-1}\right\} \\
& =\operatorname{tr}\left\{\left[\sum_{j \in \mathcal{A}_{i}^{L}} \frac{\lambda_{i j}}{N_{0} / 2+\boldsymbol{a}_{i}^{T} \boldsymbol{p}^{J}} \boldsymbol{\phi}_{i j} \boldsymbol{\phi}_{i j}^{T}\right]^{-1}\right\} \\
& =\left(N_{0} / 2+\boldsymbol{a}_{i}^{T} \boldsymbol{p}^{J}\right) \operatorname{tr}\left\{\left[\sum_{j \in \mathcal{A}_{i}^{L}} \lambda_{i j} \boldsymbol{\phi}_{i j} \boldsymbol{\phi}_{i j}^{T}\right]^{-1}\right\} \\
& \triangleq r_{i} \boldsymbol{a}_{i}^{T} \boldsymbol{p}^{J}+r_{i} N_{0} / 2
\end{aligned}
$$

where

$$
r_{i} \triangleq \operatorname{tr}\left\{\left[\sum_{j \in \mathcal{A}_{i}^{L}} \lambda_{i j} \phi_{i j} \phi_{i j}^{T}\right]^{-1}\right\} .
$$

Hence, $\operatorname{tr}\left\{\boldsymbol{J}_{i}\left(\boldsymbol{x}_{i}, \boldsymbol{p}^{J}\right)^{-1}\right\}$ is an affine function with respect to vector $\boldsymbol{p}^{J}$.

Lemma 3.1 states the CRLB for each target node is an affine function of the power vector of the jammer nodes. Based on this result, two approaches are proposed in the following for optimal power allocation of jammer nodes, and convex (in fact, linear) optimization problems, which can efficiently be solved, are obtained.

1) Optimal Power Allocation based on Average CRLB: In this case, the average CRLB for the target nodes is to be maximized under total and peak power constraints on the jammer nodes, which leads to the following formulation:

$$
\begin{aligned}
\underset{\boldsymbol{p}^{J}}{\operatorname{maximize}} & \frac{1}{N_{T}} \sum_{i=1}^{N_{T}} \operatorname{tr}\left\{\boldsymbol{J}_{i}\left(\boldsymbol{x}_{i}, \boldsymbol{p}^{J}\right)^{-1}\right\} \\
\text { subject to } & \mathbf{1}^{T} \boldsymbol{p}^{J} \leq P_{T} \\
& 0 \leq P_{\ell}^{J} \leq P_{\ell}^{\text {peak }}, \quad \ell=1,2, \ldots, N_{J}
\end{aligned}
$$

where $P_{T}$ is the total available jammer power and $P_{\ell}^{\text {peak }}$ is the maximum allowed power (peak power) for jammer $\ell$. From (14), the problem in (16) can be expressed as a linear programming (LP) problem as follows [21]:

$$
\begin{aligned}
\underset{\boldsymbol{p}^{J}}{\operatorname{maximize}} & \left(\sum_{i=1}^{N_{T}} r_{i} \boldsymbol{a}_{i}^{T}\right) \boldsymbol{p}^{J} \\
\text { subject to } & \mathbf{1}^{T} \boldsymbol{p}^{J} \leq P_{T} \\
& 0 \leq P_{\ell}^{J} \leq P_{\ell}^{\text {peak }}, \quad \ell=1,2, \ldots, N_{J}
\end{aligned}
$$

where the scaling term $1 / N_{T}$ and the constant term $\left(N_{0} / 2\right) \sum_{i=1}^{N_{T}} r_{i}$ are omitted since they have no effects on the optimal value of the power vector of the jammer nodes.

The following theorem presents the solution of (17):

Theorem 3.2: Define $\boldsymbol{w} \triangleq \sum_{i=1}^{N_{T}} r_{i} \boldsymbol{a}_{i}$, and let $h(j)$ denote the index of the $j$ th largest element of vector $\boldsymbol{w}$, 
where $j=1, \ldots, N_{J} \cdot{ }^{2}$ Then, the elements of the optimal solution $\boldsymbol{p}_{\mathrm{opt}}^{J}$ of (17) can be expressed as

Scheme 1:

$$
\boldsymbol{p}_{\mathrm{opt}}^{J}(h(j))=\min \left\{P_{T}-\sum_{l=1}^{j-1} \boldsymbol{p}_{\mathrm{opt}}^{J}(h(l)), P_{h(j)}^{\text {peak }}\right\}
$$

for $j=1, \ldots, N_{J}$, where $\boldsymbol{p}_{\mathrm{opt}}^{J}(h(j))$ represents the $h(j)$ th element of $\boldsymbol{p}_{\text {opt }}^{J}$, and $\sum_{l=1}^{0}(\cdot)$ is defined as zero.

Proof: First it is observed that the elements of $\boldsymbol{w}$ defined in the theorem are all positive, which is based on the definitions of $\boldsymbol{a}_{i}$ and $r_{i}$ in (10) and (15), respectively. ${ }^{3}$ In addition, from the definition of $\boldsymbol{w}$, the objective function in (17) can be expressed as $\boldsymbol{w}^{T} \boldsymbol{p}^{J}$. Then, under the constraints in (17), $\boldsymbol{w}^{T} \boldsymbol{p}^{J}$ can be maximized by assigning the maximum allowed power (i.e., $\min \left\{P_{T}, P_{h(1)}^{\text {peak }}\right\}$ ) to the jammer node corresponding to the largest element of $\boldsymbol{w}$ (that is, the $h(1)$ th element), the remaining power (subject to the peak power constraint) to the jammer node corresponding to the second largest element of $\boldsymbol{w}$ (that is, the $h(2)$ th element), and so on. Hence, the solution in (18) can be obtained.

2) Optimal Power Allocation based on Minimum CRLB: Another approach is to design the power allocation strategy of the jammer nodes in order to maximize the best accuracy (i.e., the minimum CRLB) of the target nodes, which leads to the following formulation:

$$
\begin{array}{cl}
\underset{\boldsymbol{p}^{J}}{\operatorname{maximize}} & \min _{i \in\left\{1,2, \ldots, N_{T}\right\}} \operatorname{tr}\left\{\boldsymbol{J}_{i}\left(\boldsymbol{x}_{i}, \boldsymbol{p}^{J}\right)^{-1}\right\} \\
\text { subject to } & \mathbf{1}^{T} \boldsymbol{p}^{J} \leq P_{T} \\
& 0 \leq P_{\ell}^{J} \leq P_{\ell}^{\text {peak }}, \quad \ell=1,2, \ldots, N_{J}
\end{array}
$$

Based on (14) and (15), the problem in (19) in the epigraph form can be expressed as the following LP problem after some manipulations [21]:

\section{Scheme 2:}

$$
\underset{\boldsymbol{p}^{J}, s}{\operatorname{maximize}} s
$$$$
\text { subject to } s-r_{i} \boldsymbol{a}_{i}^{T} \boldsymbol{p}^{J}-r_{i} \frac{N_{0}}{2} \leq 0, i=1,2, \ldots, N_{T}
$$

$$
\begin{aligned}
& \mathbf{1}^{T} \boldsymbol{p}^{J} \leq P_{T} \\
& 0 \leq P_{\ell}^{J} \leq P_{\ell}^{\text {peak }}, \ell=1,2, \ldots, N_{J}
\end{aligned}
$$

The following result presents a feature of the optimal solution for Scheme 2:

Theorem 3.3: Assume that $P_{T}<\sum_{\ell=1}^{N_{J}} P_{\ell}^{\text {peak }}$. Then, the optimal solution of (19) (equivalently, (20)) always operates at the total power limit; that is, $\mathbf{1}^{T} \boldsymbol{p}_{\mathrm{opt}}^{J}=P_{T}$.

Proof: Consider a power allocation strategy denoted by $\boldsymbol{p}_{*}^{J}$ such that $\mathbf{1}^{T} \boldsymbol{p}_{*}^{J}<P_{T}$. Then, based on the

${ }^{2}$ For example, if $\boldsymbol{w}=\left[\begin{array}{lllll}2 & 5 & 1 & 3 & 2\end{array}\right]^{T}$, then $h(1)=2, h(2)=4$, $h(3)=1, h(4)=5$, and $h(5)=3$.

${ }^{3}$ Note from (14) and (15) that the CRLB in the absence of jammer nodes (that is, for $\boldsymbol{p}^{J}=\mathbf{0}$ in (14)) is given by $r_{i} N_{0} / 2$, which is a positive quantity. assumption in the theorem, at least one power level, say the $k$ th one, should be strictly lower than its peak power limit; that is, $\boldsymbol{p}_{*}^{J}(k)<P_{k}^{\text {peak }}$, where $\boldsymbol{p}_{*}^{J}(k)$ denotes the $k$ th element of $\boldsymbol{p}_{*}^{J}$. Then, consider another power allocation strategy represented by $\boldsymbol{p}_{+}^{J}$, which is defined as $\boldsymbol{p}_{+}^{J}(j)=\boldsymbol{p}_{*}^{J}(j)$ for $j \in\left\{1, \ldots, N_{J}\right\} \backslash\{k\}$ and $\boldsymbol{p}_{+}^{J}(k)=\min \left\{\boldsymbol{p}_{*}^{J}(k)+P_{T}-\mathbf{1}^{T} \boldsymbol{p}_{*}^{J}, P_{k}^{\text {peak }}\right\}$. Namely, strategy $\boldsymbol{p}_{+}^{J}$ uses the same power levels as strategy $\boldsymbol{p}_{*}^{J}$ for all the jammer nodes except for the $k$ th one for which it employs a higher power level. Then, the objective function in (19) can be shown to be higher for $\boldsymbol{p}_{+}^{J}$ than that for $\boldsymbol{p}_{*}^{J}$ as follows:

$$
\begin{aligned}
& \min _{i \in\left\{1,2, \ldots, N_{T}\right\}} \operatorname{tr}\left\{\boldsymbol{J}_{i}\left(\boldsymbol{x}_{i}, \boldsymbol{p}_{+}^{J}\right)^{-1}\right\} \\
& =\min _{i \in\left\{1,2, \ldots, N_{T}\right\}}\left(r_{i} \boldsymbol{a}_{i}^{T} \boldsymbol{p}_{+}^{J}+r_{i} N_{0} / 2\right) \\
& >\min _{i \in\left\{1,2, \ldots, N_{T}\right\}}\left(r_{i} \boldsymbol{a}_{i}^{T} \boldsymbol{p}_{*}^{J}+r_{i} N_{0} / 2\right) \\
& =\min _{i \in\left\{1,2, \ldots, N_{T}\right\}} \operatorname{tr}\left\{\boldsymbol{J}_{i}\left(\boldsymbol{x}_{i}, \boldsymbol{p}_{*}^{J}\right)^{-1}\right\}
\end{aligned}
$$

where (14) is employed in obtaining the equalities in (22) and (24), and (23) follows from the facts that $\boldsymbol{a}_{i} \succ \mathbf{0}$ for $i \in\left\{1,2, \ldots, N_{T}\right\}$ and $\boldsymbol{p}_{+}^{J}$ is the same as $\boldsymbol{p}_{*}^{J}$ for all the entries except for the $k$ th one for which it is larger. Based on (21)-(24), it is observed that $\boldsymbol{p}_{+}^{J}$ achieves a larger minimum CRLB than $\boldsymbol{p}_{*}^{J}$, which corresponds to an arbitrary strategy that does not utilize the total available jammer power. Hence, it is concluded that any power allocation strategy that does not operate at the total power limit $P_{T}$ cannot be optimal.

It is noted from (18) and (20) that the computational complexity of the proposed optimal power allocation strategies is quite low in general.

\section{Simulation Results}

In this section, performance of the proposed schemes (Scheme 1 and Scheme 2) is evaluated through computer simulations. Since there exists no previous work on optimal power allocation for jammer nodes in a wireless localization system, the proposed schemes are compared with uniform power allocation in order to provide intuitive explanations. The uniform power allocation scheme (named Uni-Scheme in the following) assigns equal power levels to all the jammer nodes; that is, $P_{\ell}^{J}=P_{T} / N_{J}$ for $\ell=1, \ldots, N_{J}$, under the assumption that $P_{\ell}^{\text {peak }}>P_{T} / N_{J}, \forall \ell \in\left\{1, \ldots, N_{J}\right\}$. For the simulations, a network consisting of four anchor nodes, three target nodes, and three jammer nodes is considered, where the node locations are as illustrated in Fig. 1. It is assumed that each target node has LOS connections to all the anchor nodes. In order to provide a simple and clear comparison of the different power allocation schemes, the total power $P_{T}$ is normalized as $\bar{P}_{T}=2 P_{T} / N_{0}$ and it is assumed that $\lambda_{i j}$ in (9) is given by $\lambda_{i j}=100 N_{0}\left\|\boldsymbol{x}_{i}-\boldsymbol{y}_{j}\right\|^{-2} / 2$; that is, the free space propagation model is considered as in [10]. 


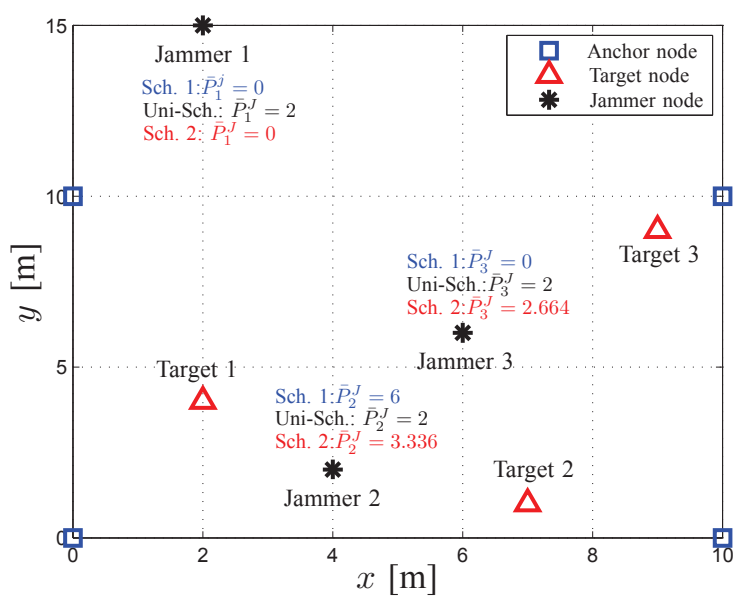

Fig. 1. The network considered in the simulations, where the anchor node positions are [0 0], [10 0], [0 10], and [ 1010 10 m., the target node positions are [2 4$]$, [ [ $\left.\begin{array}{ll}7 & 1\end{array}\right]$, and [ [9 9 ] m., and the jammer node positions are [2 15], [4 2], and [ 66$] \mathrm{m}$. Allocated powers to jammer nodes for different schemes are shown in the figure for $\bar{P}_{T}=6$.

It is also assumed that $\left|\gamma_{i j}\right|^{2}$ in (10) is expressed as $\left|\gamma_{i j}\right|^{2}=\left\|\boldsymbol{x}_{i}-\boldsymbol{z}_{j}\right\|^{-2}$. In addition, $N_{0}$ is set to 2 , and the peak power limits are taken as $P_{\ell}^{\text {peak }}=20, \forall \ell$. Based on these settings, different schemes are compared in terms of the average, minimum, and individual CRLBs in the following.

The CRLBs of Scheme 1 in (18), Scheme 2 in (20) and Uni-Scheme are plotted in Fig. 2 and Fig. 3. In Fig. 2, the average and the minimum CRLBs are illustrated versus the normalized total power $\bar{P}_{T}$. It is observed that Scheme 1 and Scheme 2 achieve the best jamming performance in terms of the average CRLB (Fig. 2(a)) and the minimum CRLB (Fig. 2(b)), respectively, which is in accordance with the problem formulations in (16) and (19). Also, Uni-Scheme is not optimal according to either criterion in this example, and significant differences from the optimal performance are observed for large normalized total powers. In other words, the proposed schemes are efective for large total jammer powers.

In Fig. 3, the CRLBs of the three target nodes are plotted versus the normalized total power for different schemes. From the CRLB curves, different behaviors are observed for different target nodes. For example, Scheme 2 makes the individual CRLBs generally larger than those for Uni-Scheme, especially for large $\bar{P}_{T}$. However, it is noted that Scheme 1 aims to degrade the average (equivalently, total) CRLB, meaning that the individual CRLBs may not be always larger than those for Uni-Scheme. The power levels of the different jammer nodes according to Scheme 1, Scheme 2, and Uni-Scheme are shown in Fig. 1 for $\bar{P}_{T}=6$. It is observed that Scheme 1 assigns all the power to jammer node 2, which is in accordance with (18). On the other hand, Scheme 2 divides the whole power between jammer node 2 and jammer node 3 , as marked in the figure.

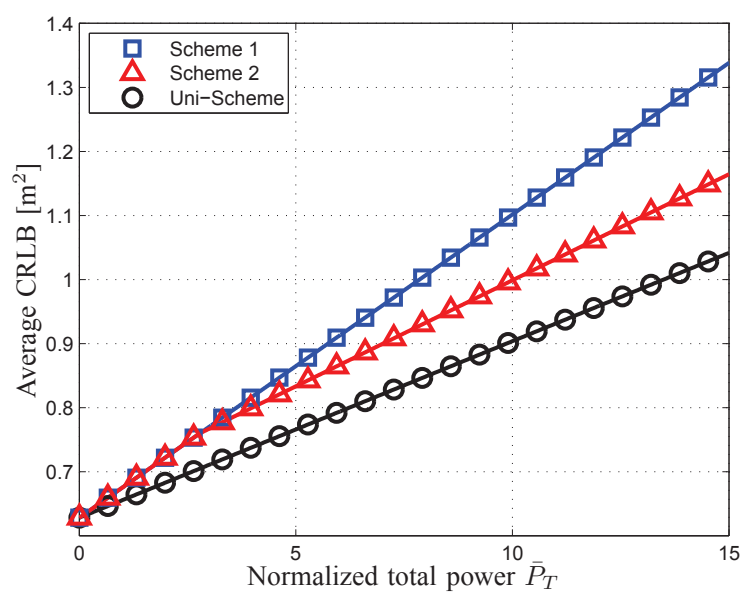

(a)

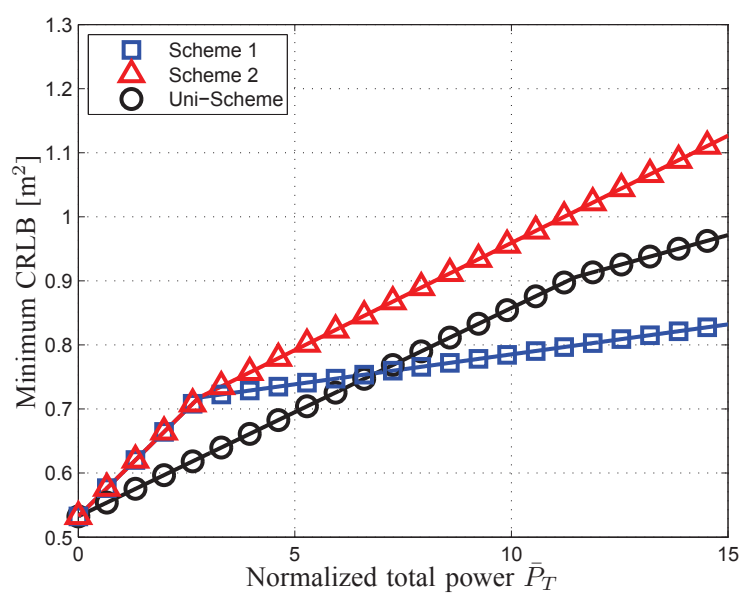

(b)

Fig. 2. Comparison of different schemes for power allocation in terms of (a) average CRLB, (b) minimum CRLB.

\section{Concluding Remarks}

In this study, optimal jamming of wireless localization systems has been investigated. Considering the CRLB on position estimation accuracy, two different schemes have been proposed to maximize certain functions of the CRLBs of the target nodes. In the first approach, power levels are allocated to jammer nodes in order to maximize the average CRLB of the target nodes whereas in the second approach the power allocation to jammer nodes is performed for the aim of maximizing the minimum CRLB of the target nodes. Both techniques have been formulated as linear programs, and a closedform expression has been obtained for the average CRLB maximization problem. In addition, the full total power utilization property has been presented for the minimum CRLB maximization problem. Simulation results have shown promising performance of the proposed techniques with respect to the uniform power allocation scheme. 


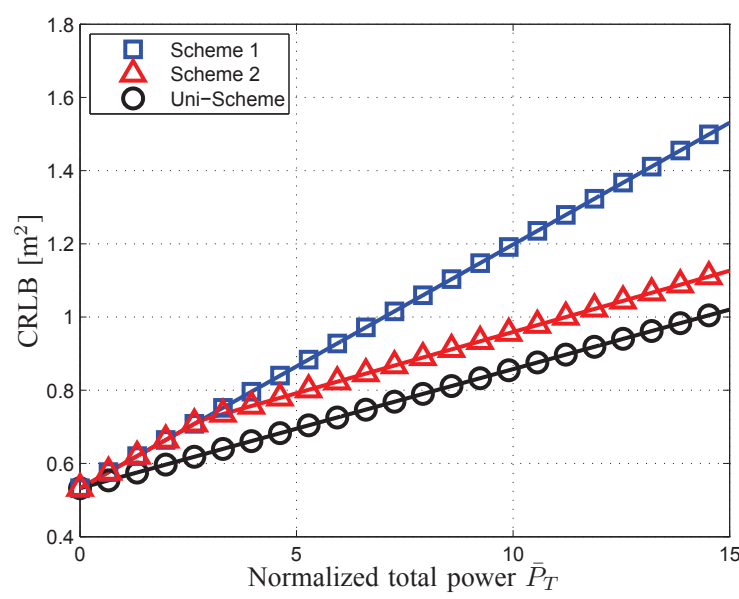

(a)

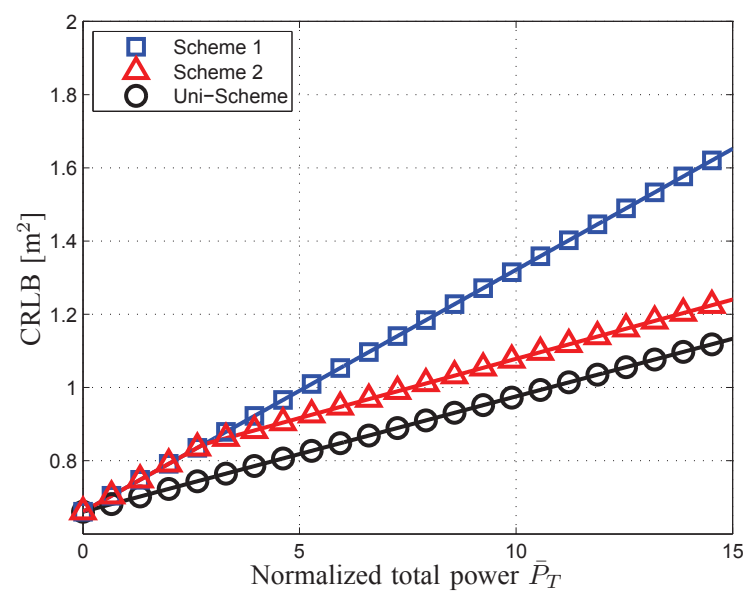

(b)

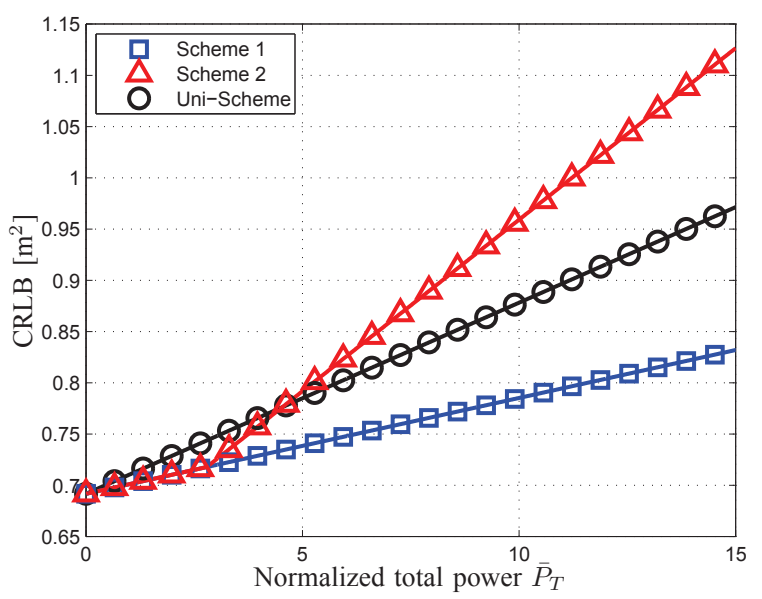

(c)

Fig. 3. CRLBs for different schemes of power allocation for (a) Target 1, (b) Target 2, and (c) Target 3 shown in Fig. 1.

\section{REFERENCES}

[1] R. Zekavat and R. M. Buehrer, Handbook of Position Location: Theory, Practice and Advances. John Wiley \& Sons, 2011.

[2] J. Figueiras and S. Frattasi, Mobile Positioning and Tracking: From Conventional to Cooperative Techniques. John Wiley \& Sons, West Sussex, 2010.

[3] Z. Sahinoglu, S. Gezici, and I. Guvenc, Ultra-Wideband Positioning Systems: Theoretical Limits, Ranging Algorithms, and Protocols. New York, Cambridge University Press, 2008.

[4] S. Gezici, "A survey on wireless position estimation," Wireless Personal Communications, vol. 44, no. 3, pp. 263-282, Feb. 2008.

[5] Y. Shen and M. Z. Win, "Fundamental limits of wideband localizationpart I: A general framework," IEEE Transactions on Information Theory, vol. 56, no. 10, pp. 4956-4980, Oct. 2010.

[6] G. Zanca, F. Zorzi, A. Zanella, and M. Zorzi, "Experimental comparison of RSSI-based localization algorithms for indoor wireless sensor networks," in Proceedings of the Workshop on Real-World Wireless Sensor Networks (REALWSN), Glasgow, U.K., Apr. 2008.

[7] H. Hu and N. Wei, "A study of GPS jamming and anti-jamming," in 2nd International Conference on Power Electronics and Intelligent Transportation System (PEITS), vol. 1, Dec. 2009, pp. 388-391.

[8] D. Lu, R. Wu, and H. Liu, "Global positioning system antijamming algorithm based on period repetitive CLEAN," IET Radar, Sonar \& Navigation, vol. 7, no. 2, pp. 1640-169, Feb. 2013.

[9] Y. Zhang and M. Amin, "Anti-jamming GPS receiver with reduced phase distortions," IEEE Signal Process. Lett., vol. 19, no. 10, pp. 635-638, Oct. 2012.

[10] W. W.-L. Li, Y. Shen, Y. J. Zhang, and M. Z. Win, "Robust power allocation for energy-efficient location-aware networks," IEEE/ACM Trans. Netw., vol. 21, no. 6, pp. 1918-1930, Dec. 2013.

[11] Y. Shen and M. Win, "Energy efficient location-aware networks," in IEEE International Conference on Communications (ICC), May 2008, pp. 2995-3001.

[12] H. Godrich, A. Petropulu, and H. Poor, "Power allocation strategies for target localization in distributed multiple-radar architectures," IEEE Trans. Signal Process., vol. 59, no. 7, pp. 3226-3240, July 2011.

[13] T. Wang, G. Leus, and L. Huang, "Ranging energy optimization for robust sensor positioning based on semidefinite programming," IEEE Trans. Signal Process., vol. 57, no. 12, pp. 4777-4787, Dec. 2009.

[14] S. Bayram, N. Vanli, B. Dulek, I. Sezer, and S. Gezici, "Optimum power allocation for average power constrained jammers in the presence of non-Gaussian noise," IEEE Commun. Lett., vol. 16, no. 8, pp. 1153-1156, Aug. 2012.

[15] T. Zhang, A. Molisch, Y. Shen, Q. Zhang, and M. Win, "Joint power and bandwidth allocation in cooperative wireless localization networks," in IEEE Conference on Communications (ICC), June 2014, pp. 2611-2616.

[16] M. K. Simon, J. K. Omura, R. A. Scholtz, and B. K. Levitt, Spread Spectrum Communications. Rockville, MD: Comput. Sci. Press, 1985.

[17] M. Weiss and S. C. Schwartz, "On optimal minimax jamming and detection of radar signals," IEEE Trans. Aeros. Elect. Sys., vol. AES-21, no. 3, pp. 385-393, May 1985.

[18] R. J. McEliece and W. E. Stark, "An information theoretic study of communication in the presence of jamming," in Int. Conf. Commun. (ICC'81), vol. 3, 1981, p. 45.

[19] Y. Qi and H. Kobayashi, "Cramér-Rao lower bound for geolocation in non-line-of-sight environment," in IEEE International Conference on Acoustics, Speech, and Signal Processing (ICASSP), vol. 3, May 2002, pp. III-2473-III-2476.

[20] S. Gezici, Z. Tian, G. B. Giannakis, H. Kobayashi, A. F. Molisch, H. V. Poor, and Z. Sahinoglu, "Localization via ultra-wideband radios: A look at positioning aspects for future sensor networks," IEEE Signal Process. Mag., vol. 22, no. 4, pp. 70-84, Jul. 2005.

[21] S. Boyd and L. Vandenberghe, Convex Optimization. Cambridge University Press, 2004. 